\title{
CORONAVIRUS DISRUPTS THE UNITED STATES ECONOMY: A CONCEPTUAL INVESTIGATION
}

\author{
Andrew N. Mason, Juntendo University, Japan
}

dx.doi.org/10.18374/JIBE-20-3.6

\begin{abstract}
This research explores economic changes resulting from the COVID-19 pandemic with a cross-disciplinary review of healthcare and economic literature. More specifically, this conceptual research provides a preliminary examination of the effects of COVID-19 on United States (US) macroeconomic activity. The uniqueness of this healthcare pandemic event results in original findings. Secondary data, acquired from the US Federal Reserve Economic Database (FRED), was used to evaluate how economic activity changed after the COVID-19 pandemic was declared in the US. Results indicate that the pandemic caused dramatic harm to major US macroeconomic indicators including GDP, unemployment, and consumer spending. Thus, the COVID-19 pandemic may be a catalyst for structural changes in the US economy.
\end{abstract}

Keywords: Coronavirus, COVID-19, US Economy, Pandemic Costs 